\title{
CONTROL DE EMOCIONES: CLAVE PARA DECISIONES EXITOSAS
}

\section{EMOTION CONTROL: KEY TO SUCCESSFULL DECISIONS}

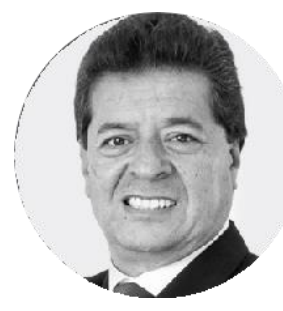

\author{
Jorge A. Villavicencio Araníbar \\ Código ORCID: 0000-0003-4915-8530 \\ Profesor de Dirección Estratégica \\ Director de TQP SAC \\ Correo electrónico: jorge.villavicencio23@gmail.com \\ Recibido: 08 de agosto de 2019 \\ Aprobado: 17 de setiembre de 2019 \\ Cómo citar este artículo: \\ Villavicencio, J. (2019). Control de emociones: clave para \\ decisiones exitosas. Review of Global Management, 5(2), 44-57.
}

Desafío Aristotélico: "Cualquiera puede ponerse furioso... eso es fácil. Pero estar furioso con la persona correcta, en la intensidad correcta, en el momento correcto, por el motivo correctoydelaforma correcta......esonoesfácil”.

\section{RESUMEN}

Las tendencias del management moderno, nos han permitido comprobar de forma certera, que uno de los cimientos en los cuales se basan los resultados organizacionales, son las personas y todo lo que gira alrededor de cada uno de los integrantes de un grupo humano: valores, misión, visión, ética, cultura organizacional, etc. Bajo esa lógica, para las empresas se vuelve de carácter imperativo, que el recurso humano en su conjunto desarrolle todo el potencial que tiene, en un 
ambiente propicio e idóneo, para el logro de los objetivos que la organización se plantea; pero es más importante aún, que estas personas, se conozcan internamente, para que, a través de las llamadas "habilidades blandas", faciliten esa consecución de objetivos. El presente ensayo pretende enfocar estos aspectos, desde una óptica que combine el carácter teórico que sustenta el conocimiento de las emociones, con propuestas que se irán planteando a raíz de la experiencia vivida en la práctica empresarial.

Palabras clave: Emociones, inteligencia emocional, empatía, valores, asertividad, automotivación.

\section{ABSTRACT}

Modern management shows that one of the foundations on which organizational results rest is people, and everything that surrounds them within a group: values, mission, vision, organizational culture, ethics, etc. Under this logic, for companies, it becomes imperative that their human resources, as a whole, develop all the potential they have, in a favorable and ideal environment, for the achievement of the objectives that the organization sets; but it is even more important that these people get to know each other internally, so that, through the socalled "soft skills", they facilitate this achievement of objectives. This essay focuses on these issues, combining theoretical arguments on emotions, with proposals based on actual professional work experience.

Keywords: Emotions, emotional intelligence, empathy, values, assertively, self-motivation.

\section{INTRODUCCIÓN}

De acuerdo con McClelland (1973), el ser humano tiene diferentes necesidades que cumplir a lo largo de su vida (al logro, a la afiliación y al poder/influencia) 
y para ello, las personas recibimos diferentes fuentes de motivación desde la misma creación del "self" (Gamarra, 2016). Todo este proceso motivacional, va de la mano con esa formación, adaptación, trabajo y desarrollo de las emociones a lo largo de nuestra vida; que, en palabras sencillas, nos va a llevar a formar esa personalidad que, en nuestra etapa de juventud y adultez, nos facilitará el logro del cumplimiento de nuestros objetivos o en caso contrario, nos propondrá una serie de trabas, las cuales debemos trabajar para superarlas y alinearnos con lo que realmente demanda nuestra propia visión de las cosas.

Muchas veces no les damos la importancia del caso, o no las ubicamos en la magnitud o escalafón que debieran, pero las emociones son aquellos mecanismos que nos ayudan a actuar de forma rápida cuando se activa algún sensor de nuestro cerebro ante un impulso dado (Fernández-Abascal y Palmero, 1999). Todos, en algún momento de nuestra vida, hemos sentido emociones de diferente tipo, como el miedo, la sorpresa, la aversión, la ira, la tristeza, la alegría), pero vemos también que todos somos completamente diferentes en términos de respuesta ante cada una de ellas. Entonces surge la interrogante siguiente: ¿realmente se pueden manejar las emociones?

Gardner (1987), indica lo siguiente: "[l]a teoría de las inteligencias múltiples involucra aquellas capacidades distintas e independientes que tienen los seres humanos, para resolver problemas o elaborar productos que sean valiosos en su cultura u otras, en cada una de las áreas que existen (lógico-matemática, musical, corporal cinética, lingüística, espacial, naturalista, interpersonal e intrapersonal)”. Bajo este contexto, podríamos afirmar que todos estamos en capacidad de desarrollar diferentes habilidades en diferentes campos, dependiendo de nuestra afinidad o interés con algunos de ellos. Destacan en el ámbito interpersonal, la autoconciencia o habilidad para saber qué está pasando en nuestro cuerpo y qué estamos sintiendo; el control emocional que permite regular la manifestación de una emoción y la capacidad de automotivarse o motivar a los demás. En el ámbito intrapersonal, podemos mencionar a la empatía y las habilidades sociales, como la popularidad, el liderazgo y la eficacia interpersonal). 
Goleman (1995), explica que "el éxito de una persona no depende del intelecto o de sus estudios académicos, sino de su inteligencia emocional; que, según el autor, es la capacidad de reconocer los sentimientos propios y los de los demás, para así manejar bien las emociones y tener relaciones más productivas con quienes nos rodean". Esta lógica nos hace pensar que cualquier individuo, con la experiencia que va ganando en el tiempo, va creciendo en el desarrollo de sus sentimientos, de tal forma que va cultivando la fortaleza emocional necesaria para afrontar las exigencias que el mundo moderno propone y obtener los resultados que nosotros y los que nos rodean esperamos.

López y Arango (2002), afirman que "la inteligencia emocional es la capacidad de dominar las emociones y configurar rasgos de carácter como la autodisciplina, la compasión y el altruismo. Consiste en saber reconocer lo que se siente, controlar las respuestas emocionales, aprender a automotivarse y a entusiasmarse con lo que se quiere. Definir y afrontar metas, afrontar la vida con confianza y optimismo, comprender los sentimientos de los demás y relacionarse armoniosamente con ellos" (pag.198). Muchas veces nos sucede que interactuamos con personas llenas de energía positiva, que, con solo saludarlas, notamos una sensación diferente hacia lo bueno de la vida. No interesa que se esté pasando por un momento difícil, complicado o cargado, estas personas nos trasmiten cordialidad, buena actitud y ganas de salir adelante.

A mi juicio, la teoría que ha desarrollado Daniel Goleman acerca de la inteligencia emocional, en las diferentes publicaciones y libros que ha escrito, son la fuente principal de inspiración para poder afirmar que actualmente, el gran reto que se tiene, es lograr ese autocontrol que nos diferencie de los demás; esa empatía tan necesaria para entender a todos nuestros stakeholders; ese entusiasmo que contagie a todos nuestros relacionados; esa tenacidad y perseverancia que nos facilita lograr metas sin interesar las barreras que siempre existen; y sobre todo, esa auto motivación que nos permitirá ir mucho más allá de lo que muchos consideran imposible de lograr. 
De alguna manera, estas pautas que nos presenta Goleman nos orientan a la identificación de aquellas emociones que son vitales para los resultados futuros y sobre las cuales debemos centrar nuestro esfuerzo para su adecuado manejo, adaptación y trabajo de mejora. Soy un convencido que la decisión está en uno mismo y la propuesta que nosotros nos hagamos sobre cómo balancear nuestras emociones para conseguir mejoras sustanciales, nos podría deparar sorpresas que muchas veces ni siquiera esperábamos.

También soy un creyente que, en el devenir de las experiencias profesionales y el crecimiento que uno tiene en el tiempo, los momentos difíciles, complicados, o de crisis, son los que nos ponen más a prueba si estamos formando nuestra personalidad de acuerdo a lo que deseamos o quizás nos estamos dejando llevar por las coyunturas y no nos estamos preparando para dar ese "gran salto".

Prati et al. (2003), mencionaron que "la inteligencia emocional refleja la habilidad de leer y entender a otros, en contextos sociales, para detectar matices de reacciones emocionales y utilizar tal conocimiento para influir en otros, a través de la regulación y el control emocional". Podemos resumir estos criterios en que un líder emocionalmente inteligente, inducirá a los miembros de su equipo a la motivación colectiva, usando una autoridad de orden carismático y una influencia transformacional, a fin de mejorar el desempeño del equipo y, a la vez, conducir a su equipo hacia una expresión de orden creativo e innovador.

Fischman (2012), presenta el modelo del "ciclo del éxito" que se muestra a continuación en la Figura 1: 


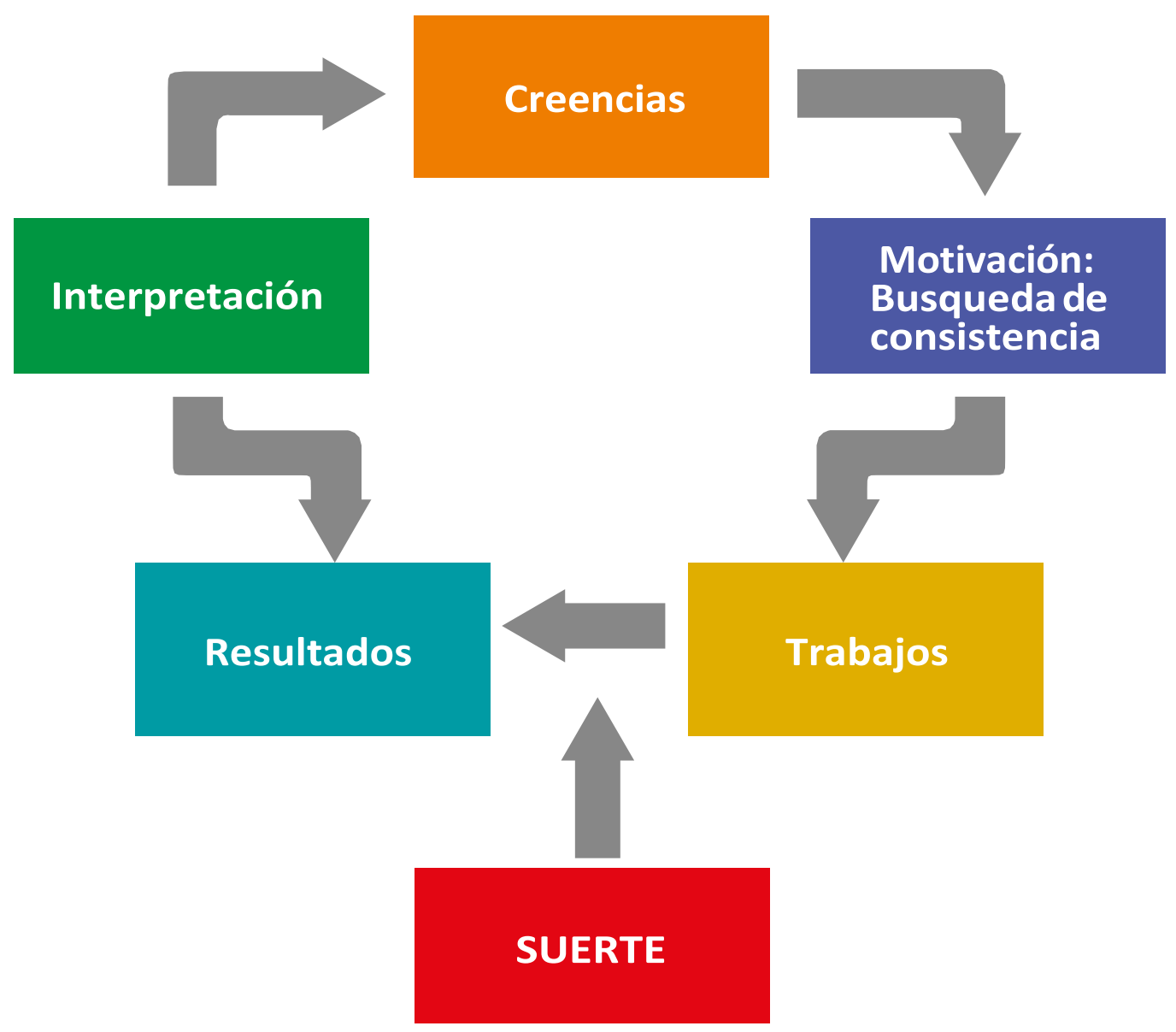

El modelo parte de las creencias que tienen las personas, positivas o negativas, y cómo estas influyen en el logro de las metas. Estas creencias tienen que ir asociadas con la automotivación que se va logrando de a pocos, trabajando con el inconsciente y arriesgando e intentando todas las veces que sea necesario, para buscar una consistencia en nuestros actos. De hecho, esto va de la mano con un trabajo arduo que nos demanda cada una de las acciones y decisiones que tomamos (nada es fácil en la vida). Como muchas cosas en la vida, uno va formando su propia suerte (a pesar de que, para muchos, esta es una acepción totalmente subjetiva), a través de su red de contactos; su intuición de las cosas; y la actitud con la que se tomen las mismas. Toda esta secuencia nos entregará resultados y lo interesante es cómo estos son interpretados, para que siempre veamos el vaso medio lleno. Así el resultado no nos acompañe en la decisión que hayamos tomado, hay que sacar la parte buena que tienen estas experiencias negativas, para regresar al ciclo del éxito con más fuerza y ganas. 
Covey (2003), establece una serie de conceptos que apoyan a que las personas sean mucho más eficientes en su vida y en la toma de decisiones, a través de lo que denomina: "7 hábitos de la gente altamente efectiva"-ver la Figura 2.

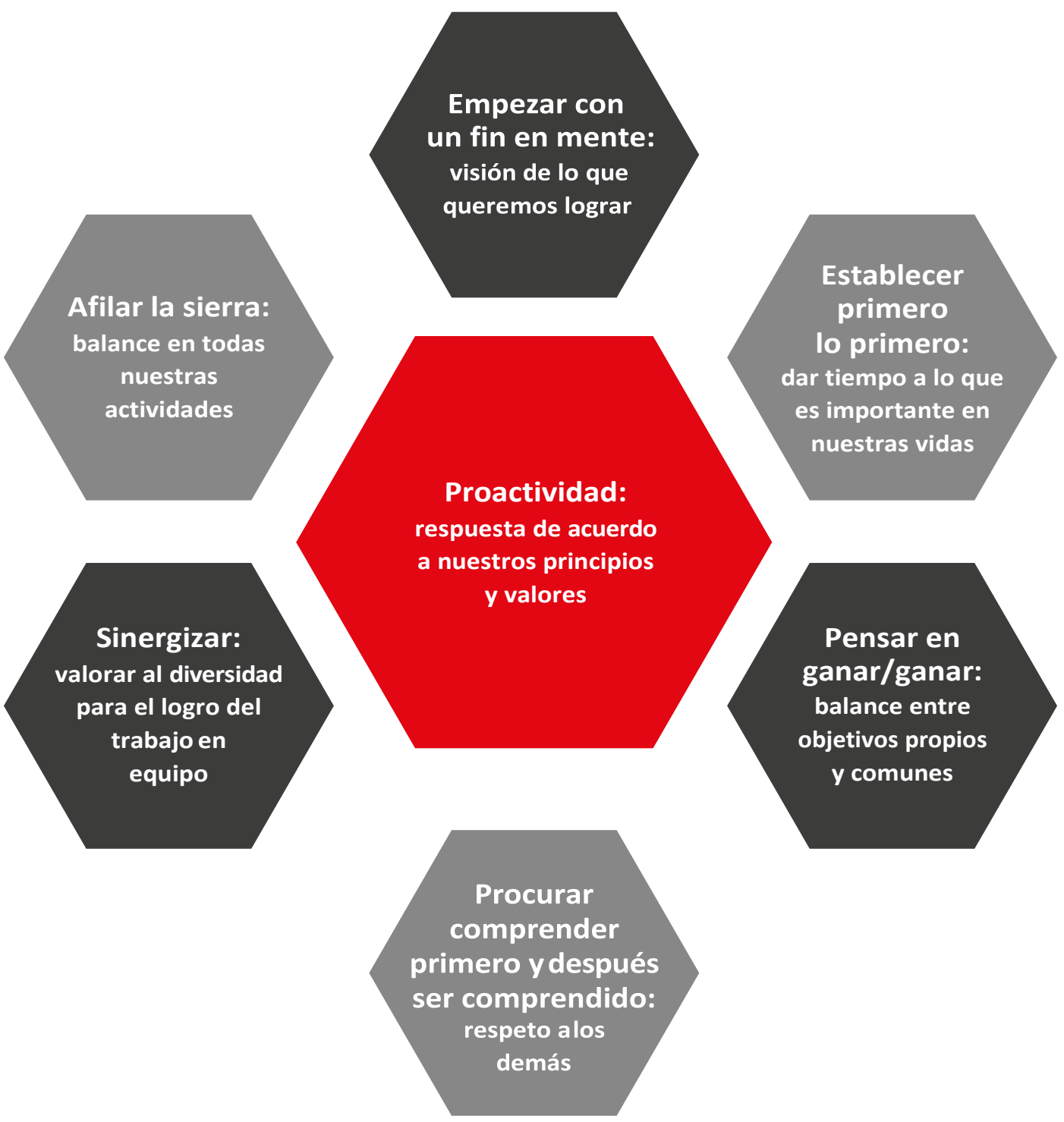


En esta propuesta se conjugan una serie de prácticas que las personas pueden ir cultivando en el tiempo, para que tengan una personalidad centrada en valores y basen su toma de decisiones en aspectos que guardan relación directa con lo que se ha comentado líneas arriba, respecto a las propuestas de Goleman y la inteligencia emocional.

\section{ANÁLISIS}

El ser humano es sumamente complejo y la influencia que recibe de su entorno directo inicialmente, desde sus primeros días de vida, hasta que comienza a interactuar con su entorno indirecto, hace que su personalidad vaya formándose y alineándose a todo aquello que considera que es bueno o malo, según esas influencias comentadas. Es por ello que reviste mayor importancia y relevancia que esos insumos iniciales estén basados en valores y creencias con un alto ingrediente ético, como de principios morales que puedan sustentar en el tiempo, todo lo que esa persona pueda evaluar y discernir para tomar las decisiones correctas en su vida.

Un factor igualmente relevante para aquellos que van a tener la gran responsabilidad de conducir gente, tiene que ver con el adecuado trato que estos subordinados reciben de su líder y es aquí donde entran a tallar una serie de conceptos y definiciones que se han revisado líneas arriba y que resaltaremos y afinaremos a continuación.

\section{INTELICENCIA EMOCIONAL}

El estudio de Goleman (2000), nos permite resumir este concepto en 
cuatro competencias: i) consciencia en sí mismo (emocional, autoevaluación y autoconfianza); ii) autodominio (autocontrol, honradez, adaptabilidad, orientación al logro e iniciativa); iii) consciencia social (empatía y orientación al servicio); y iv) habilidades sociales (liderazgo visionario, influencia, comunicación, manejo de conflictos, trabajo en equipo y colaboración). Resulta claramente relevante que la formación y el cultivo de estas características claves, nos van a permitir enfrentar las diferentes exigencias que nos presenta la vida; con una mayor sapiencia, entereza y balance, tanto para nuestros propios ideales, como para quienes, en mayor o menor grado, tienen relación directa o indirecta con las decisiones que vamos tomando.

\section{LIDERAZCO}

Si es verdad que existen muchos estudios sobre este tema, no existe una definición única que englobe este importante factor para que las decisiones se tomen de la mejor forma. De lo que no debemos tener la menor duda, es que un líder debe ser un individuo sumamente capaz que realice un aporte muy valioso con su talento, conocimiento, habilidades y buenos hábitos de trabajo. Asimismo, debe ser parte activa del trabajo, contribuyendo con su capacidad personal, al logro colectivo; trabajando en equipo; y organizando efectivamente a la gente. Esta persona debe saber catalizar el compromiso y perseguir vigorosamente una visión, para, de esa forma, construir un crecimiento sostenido.

\section{3. ÉXITO}

Cuando vamos teniendo un conocimiento real de las cosas, desde que somos pequeños, soñamos diferentes cosas que nos podrían pasar y por qué no decirlo, que nos gustarían que pasen. 
Como se dice también de forma coloquial "soñar no cuesta nada". Sin embargo, esa visión de lo que queremos de la vida, de nuestro futuro, de los logros, de las metas, etc., nunca va podría realizarse mientras no estemos lo suficientemente motivados y convencidos de que podemos lograrlo; de que vamos a realizar todo el esfuerzo que podamos y necesitemos para conseguir ese sueño. El trabajo arduo y sostenido es una de las reglas básicas para lograr lo que nos proponemos; nada nos va a caer del cielo y nadie nos va a regalar nada. Nosotros mismos tenemos que fijarnos el cumplimiento de lo que nos proponemos y no debemos pestañear hasta conseguirlo. Habrá situaciones que quizás no nos acompañen en términos de los resultados esperados, pero aquí también viene el concepto de resiliencia que debemos poner en práctica permanentemente, para sacar lo mejor de esas experiencias contrarias y volver a la carga con más energía y con conocimiento de causa; de que el camino que tomamos anteriormente nos ha entregado un resultado no deseado.

Dentro de todo este contexto del logro de las metas y el éxito que conllevan estos resultados, hay un factor que considero que siempre está presente en mayor o menor grado: la suerte. De hecho, es un concepto contradictorio y no hay definiciones exactas sobre el mismo, pero lo que sí es muy cierto es que cada uno va formando su propia suerte en el camino. Existen estudios realizados por Wiseman (2003) y Gunther (2009), que han tratado de comparar a gente que se considera suertuda y gente que no, obteniéndose como resultado común, ciertas características que definen a las personas con suerte: i) la gente con suerte tiene una actitud relajada hacia la vida; ii) la gente con suerte escucha su intuición; iii) la gente con suerte cree que tiene buena suerte; y iv) liderazgo

\section{HABILIDADES YATRIBUTOS}

En este acápite, vamos a poder visualizar cómo las personas pueden ser tan 
diferentes unas a otras, en función de sus destrezas y sobre todo de cómo interpretan lo que se conoce como ética del carácter: "principios básicos para vivir con efectividad". Estos principios engloban todo lo relacionado a la humildad, integridad, paciencia, esfuerzo, valor, justicia, mesura, simplicidad y modestia, entre otros. Básicamente nuestro carácter está compuesto por nuestros hábitos y atributos. "Siembra un pensamiento, cosecha una acción; siembra una acción, cosecha un hábito; siembra un hábito, cosecha un carácter; siembra un carácter, cosecha un destino", dice el proverbio. Los hábitos y los atributos centran, a su vez, tres criterios que están entrelazados entre sí: el conocimiento (el qué y el porqué de las cosas); la capacidad (el cómo); y el deseo (el querer hacerlo). El adecuado equilibrio de nuestros pensamientos, prioridades, hábitos y atributos, de alguna manera nos llevan a formar una clara distancia entre lo que forma nuestra propia independencia para tomar decisiones, para luego, siguiendo en esa senda del equilibrio, ir lograr poco a poco lo que conocemos como interdependencia con todos aquellos stakeholders que cada uno de nosotros tenemos en nuestros diferentes ámbitos de la vida personal, laboral, familiar, entre otros.

\section{COMENTARIOS FINALES}

La vida nos enfrenta a diferentes retos desde nuestros primeros años y al inicio, casi de forma casi empírica, nos obliga a tomar decisiones permanentemente. Toda la influencia que recibimos en nuestros primeros años de formación, de alguna forma, van marcando nuestro camino, en términos de personalidad, carácter y habilidades; los mismos que en el devenir de nuestras tareas cotidianas, en el ámbito personal y laboral, van acentuando esos hábitos y atributos que nos servirán para cosechar triunfos y en algunas ocasiones, quizás malas experiencias.

Todos estos factores, van a formar parte también de aquellos rasgos y perfiles de liderazgo que podemos ir ganando y trabajando en el camino, sobre todo, cuando comenzamos a tener gente bajo nuestra responsabilidad: hijos en el caso familiar y colaboradores en el caso laboral. 
La pregunta que siempre sale a la luz es si el líder nace o se hace, la misma que siempre va a generar infinidad de opiniones, divididas, que pocas veces convergen en algo único; pero lo que sí se puede afirmar es que aquellos que han sabido trabajar claramente su personalidad, carácter y sus habilidades, transformándolas en hábitos y atributos estratégicos para el éxito, son quienes mayor beneficio van a poder obtener en esta difícil tarea de conducir gente. Más aun, cuando las situaciones difíciles o de crisis se presentan sin mayor aviso, es allí cuando salen a relucir todos estos atributos mencionados, que, asociados de forma directa con la inteligencia emocional, facilitan al líder salir adelante en esos momentos críticos que requieren cabeza fría para tomar la mejor decisión posible.

Las decisiones exitosas están a la vuelta de la esquina, pero hay que trabajar mucho para que se puedan dar con la mayor naturalidad, viabilidad y simpleza posible; sobre todo en aquellos conceptos que hemos compartido en el presente ensayo, entre los cuales debo resaltar de manera particular el autocontrol y la automotivación. La sinergia y equilibrio entre ambos conceptos facilitan mucho las cosas.

En conclusión, presento algunas recetas propias que de alguna manera pueden servir para lograr un adecuado balance emocional: Pensar siempre positivamente: recordar, sobre todo, aquellas virtudes, éxitos que se han logrado y las fortalezas que han permitido crecer en la vida.

1. Si la situación lo amerita, distraer la atención hacia un asunto concreto. Esto alivia de buena forma aquella tensión que estaría por avecinarse.

2. Enfocarse en el futuro inmediato: al ser consciente que todas las decisiones conllevan una consecuencia, positiva o negativa, se debe tener en cuenta que esos resultados no duran toda la vida, tienen su momento y su espacio. 


\section{REFERENCIAS}

Covey S. (2003). Los 7 hábitos de la gente altamente efectiva. Buenos Aires (Argentina). Editorial Paidós.

Fernández-Abascal E. y Palmero F. (1999) Emociones y Salud. Barcelona (España). Ediciones Ariel.

Fischman D. (2012) El éxito es una decisión. Lima (Perú). Editorial Universidad Peruana de Ciencias Aplicadas

Gamarra Morgenstern, B., (2016) El sujeto creado. Santa Fe de Bogotá (Colombia) Editorial Kimpres SAS.

Gardner, H. (1987). Estructuras de la mente. La teoría de las inteligencias múltiples. Ciudad de México (México), Fondo de Cultura Económica.

Goleman D. (1995) Inteligencia emocional. Por qué es más importante que el cociente intelectual. España:. Ediciones B, S.A.

Goleman D. (2000). Leadership that gets results. Harvard Business Review, 78(2), 78-90.

Gunther M. (2009). The luck factor: why some people are luckier than others and how you become one of them. Hampshire: Harriman House LTD.

López E. y Arango T. (2002). Inteligencia Emocional. Aprendiendo y creciendo juntos. Bogotá (Colombia). Ediciones Gamma S.A.

McClelland, David, (1973) The Human Motivation. Cambridge University Press. 
Prati L.M., Douglas C., Ferris G.R., Ammester A.P. \& Bucley M.R. (2003). Emotional intelligence, leadership effectiveness and team outcomes. The International Journal of Organizational Analysis, 2(4), 21-40.

Wiseman R. (2003). The luck factor. The four essential principles. Nueva York: Miramax Books. 